\title{
First direct evidence of the CNO fusion cycle in the Sun with Borexino
}

\section{S. Kumaran ${ }^{a, b}$ on behalf of the Borexino Collaboration}

(a complete list of authors can be found at the end of the proceedings)

${ }^{a}$ Forschungszentrum Jülich - Institute for Nuclear Physics, IKP-2, Jülich, Germany

${ }^{b}$ RWTH Aachen University - Physics Institute III B, Aachen, Germany

E-mail: sindhujha.kumaran@rwth-aachen.de

The Borexino experiment has recently provided the first direct experimental evidence of the subdominant CNO-cycle in the Sun, which is assumed to be the main energy production mechanism in heavier stars. Borexino is a liquid scintillator detector located at the Laboratori Nazionali del Gran Sasso, Italy with the main goal to measure solar neutrinos. The extreme radiopurity of the scintillator and the successful thermal stabilisation of the detector have proven to be valuable assets in the quest for $\mathrm{CNO}$ neutrinos. The low abundance of $\mathrm{CNO}$ neutrinos and the similarity of its spectral shape to that of pep solar neutrinos and the intrinsic ${ }^{210} \mathrm{Bi}$ background, make $\mathrm{CNO}$ neutrino detection challenging. Therefore, it is necessary to constrain these backgrounds independently. The energy and radial distribution of the events can then be exploited to perform a multivariate fit, which requires a careful evaluation of the systematic uncertainty associated with the Monte-Carlo spectral shapes used. Borexino has successfully rejected the null hypothesis of CNO-cycle neutrinos in the Sun with greater than 5.0 $\sigma$ significance with 99\% C.L. This article will present the overview of the strategy and methods used to achieve this result and the consequence of this result for solar and stellar physics.

$37^{\text {th }}$ International Cosmic Ray Conference (ICRC 2021)

July 12th - 23rd, 2021

Online - Berlin, Germany 


\section{Solar neutrinos}

Solar neutrinos are produced copiously in the Sun due to the nuclear fusion processes that happen in its core. While the photons take around $10^{5}$ years to reach the Earth, the neutrinos only take 8 minutes, and carry direct, unperturbed information about the Sun's core. The main source of energy powering the sun and sun-like stars is the $p p$ fusion chain. The $p p$-chain reactions result in the fusion of four protons into helium.

$$
4 p \longrightarrow^{4} \mathrm{He}+2 e^{+}+2 v_{e}
$$

The process happens through different alternative chains namely, $p p$-I, $p p$-II, and $p p$-III. Each neutrino produced in the $p p$-chain is called by the name of the reaction it was produced in: $p p, p e p$, ${ }^{7} \mathrm{Be},{ }^{8} \mathrm{~B}$, and hep. The $\mathrm{CNO}$ cycle is hypothesised to be the main process in heavier stars [1], [2]. It has two sub-cycles namely, CNO-I and CNO-II. The CNO-I sub-cycle is more dominant (99.95\%) and is simply referred to as the CNO-cycle. Neutrinos produced in this cycle originate from the $\beta$ - decays of ${ }^{15} \mathrm{O}$ and ${ }^{13} \mathrm{~N}$ have continuous energy spectra with endpoints at around $1.5 \mathrm{MeV}$, and $1.7 \mathrm{MeV}$, respectively. The neutrinos are labeled according to the reactions they are produced in as: ${ }^{13} \mathrm{~N},{ }^{15} \mathrm{O}$, and ${ }^{17} \mathrm{~F}$. The theoretical energy spectra of solar neutrinos is taken from [3] and shown in Figure 1.

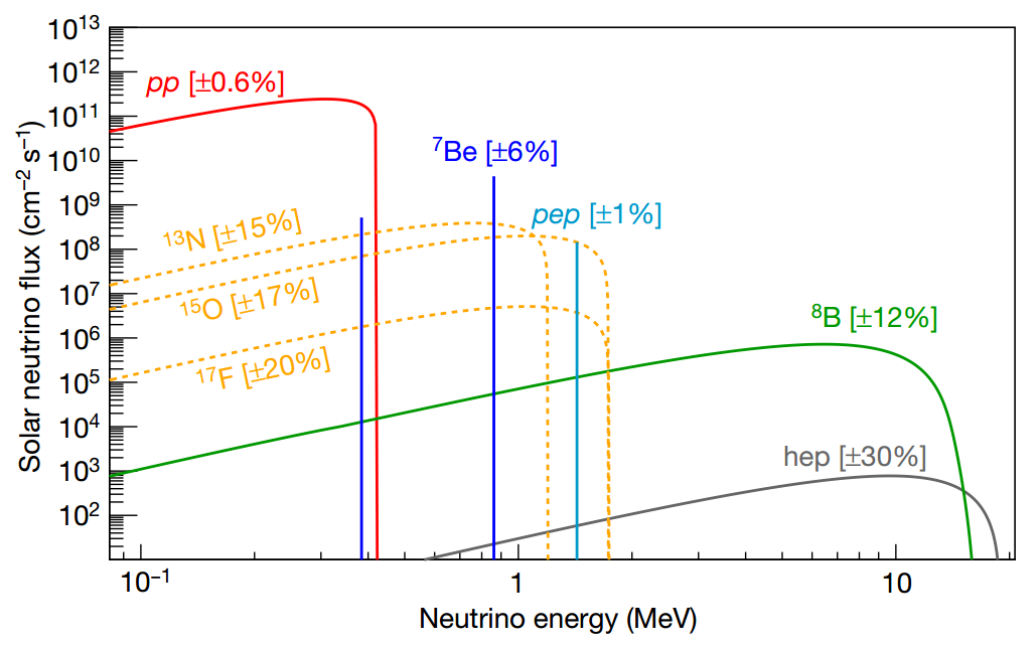

Figure 1: The theoretical energy spectra of solar neutrinos taken from [3]. The flux is given in units of $\mathrm{cm}^{-2} \mathrm{~s}^{-1} \mathrm{MeV}^{-1}$ for continuum sources and in $\mathrm{cm}^{-2} \mathrm{~s}^{-1}$ for monoenergetic sources.

\subsection{Standard solar model}

The Standard Solar Model (SSM) is based on different assumptions. It is assumed that the solar energy is produced through the $p p$-chain, contributing to $>99 \%$ of the energy, while the contribution of the CNO-cycle is yet unknown. The Sun is in a state of thermal and hydrostatic equilibrium. The solar composition is defined by its initial elemental abundance. The energy transport from the core to the surface occurs through radiation and convection. The model relies on standard solar parameters namely, luminosity measured through irradiance $\left(3.828 \times 10^{26} \mathrm{~W}\right)$, 


\begin{tabular}{|c|c|c|c|}
\hline solar $v$ & HZ SSM & LZ SSM & difference HZ-LZ (\%) \\
\hline \hline$p p$ & $5.98(1 \pm 0.006) \cdot 10^{10}$ & $6.03(1 \pm 0.005) \cdot 10^{10}$ & -0.84 \\
pep & $1.44(1 \pm 0.01) \cdot 10^{8}$ & $1.46(1 \pm 0.009) \cdot 10^{8}$ & -1.39 \\
$h e p$ & $7.98(1 \pm 0.030) \cdot 10^{3}$ & $8.25(1 \pm 0.30) \cdot 10^{3}$ & -3.38 \\
${ }^{7} \mathrm{Be}$ & $4.93(1 \pm 0.06) \cdot 10^{9}$ & $4.50(1 \pm 0.06) \cdot 10^{9}$ & +8.72 \\
${ }^{8} \mathrm{~B}$ & $5.46(1 \pm 0.12) \cdot 10^{6}$ & $4.50(1 \pm 0.12) \cdot 10^{6}$ & +17.58 \\
$\mathrm{CNO}$ & $4.88(1 \pm 0.11) \cdot 10^{8}$ & $3.51(1 \pm 0.11) \cdot 10^{8}$ & +28.07 \\
\hline
\end{tabular}

Table 1: Solar neutrino fluxes, predicted by HM- and LM-SSMs, in units of $\mathrm{cm}^{-2} \mathrm{~s}^{-1}$ [3].

age estimated through radioactive dating of meteorites, rocks, samples from Earth and Moon (4.57 $\pm 0.01 \mathrm{Gyr})$, mass computed via orbit equation of Earth $\left(1.988 \times 10^{33} \mathrm{~kg}\right)$, radius calculated based on luminosity and surface temperature $\left(6.955 \times 10^{8} \mathrm{~m}\right)$, and initial elemental abundances inferred through spectroscopy and helioseismology.

Solar metallicity: A crucial parameter related to the abundance of different elements in the Sun, is the highly debated solar metallicity i.e. the fraction of elements heavier than helium, called metals in the context of solar physics. It is denoted by $(\mathrm{Z} / \mathrm{X}) \odot$, where $\mathrm{Z}$ is the abundance of metals and $\mathrm{X}$ is the abundance of protons and Helium in the Sun. Solar metallicity provides answers about the neutrino fluxes and the speed of sound waves in the Sun. The newest generation of SSMs, called B16 [3], employs two central sets of solar abundances, based on photospheric and meteoritic abundances from different inputs, and results in different predictions of solar metallicity. The GS98 is called the High metallicity (HZ) SSM [4], and the AGSS09met is called the Low metallicity (LZ) SSM [5], predicting a $(\mathrm{Z} / \mathrm{X}) \odot$ value of 0.0229 and 0.0178 , respectively. The different ratios lead to different predictions of solar neutrino fluxes as shown in Table 1. It can also be observed that the experimental measurements of ${ }^{7} \mathrm{Be},{ }^{8} \mathrm{~B}$, and $\mathrm{CNO}$ neutrino fluxes can provide hints towards metallicity since the neutrinos come from metals heavier than Helium.

\section{The Borexino experiment}

Borexino is an ultra-pure liquid scintillator detector, located underground at the Laboratori Nazionali del Gran Sasso in Italy [6], with the main goal to measure solar neutrinos. It is placed at 3800 metres water equivalent depth where the cosmic muon flux is suppressed by a factor of around $10^{6}$. The active core of the detector consists of approximately $280 \mathrm{t}$ of liquid scintillator contained in a spherical nylon vessel with a radius of $4.25 \mathrm{~m}$. Particles that interact in the scintillator emit light, which is detected by 2212 photomultiplier tubes. Solar neutrinos are detected by Borexino via their elastic scattering off electrons. The total number of detected photons and their arrival times are used to reconstruct the electron recoil energy and the interaction point in the detector, respectively. The energy (E) and spatial resolution $(\sigma)$ of Borexino has slowly deteriorated over time owing to the steady loss of photomultiplier tubes (on average 1238 active channels for this analysis), with current values of $\left.\sigma_{E} / \sqrt{(} E\right) \approx 6 \%$ and $\sigma_{x, y, z} \approx 11 \mathrm{~cm}$ for $1 \mathrm{MeV}$ events at the centre of the detector [7]. The success of the Borexino experiment is the result of its unprecedented radiopurity combined with the careful selection of materials and clean assembly protocols. The experiment is divided into three phases, with their beginnings corresponding to the end of major 
stabilisation operations. Borexino has already provided a complete spectroscopy of $p p$-chain solar neutrinos [8]. Phase-III of the experiment started after the thermal insulation and stabilisation of the detector, the key requirement for the CNO-neutrino detection and runs from July 2016 to February 2020, corresponding to 1,072 days of live time.

\section{CNO detection: Challenges and Strategy}

The main challenge of CNO neutrino measurement, apart form their extremely low abundance, is the similarity of spectral shapes of $\mathrm{CNO}$ neutrinos, pep neutrinos, and the intrinsic ${ }^{210} \mathrm{Bi}$ background (Figure 2 in [7]). Therefore, it is absolutely necessary to constrain the rates of pep and ${ }^{210} \mathrm{Bi}$ with the exposure of Phase-III.

The $p e p$ neutrino rate can be constrained using the theoretical constraint on the $p p / p e p$ neutrino rate, solar luminosity constraints, and the global fit of solar neutrino rate without Borexino PhaseIII, considering the MSW effect on the neutrino propagation, as well as the errors on the neutrino oscillation parameters. The processes leading to $p p$ and $p e p$ neutrinos depend on the same nuclear matrix element, thereby making their ratio independent of the SSM predictions. This ratio has been estimated in a robust way with $\sim 1 \%$ precision. The uncertainty on the pep neutrinos can be determined in this way with $\sim 10 \%$ precision. The precision can be further improved by performing a global analysis with all solar neutrino experiments, applying a luminosity constraint, and using the most recent values for the oscillation parameters. The final value obtained for the pep constraint in this way is $2.74 \pm 0.04 \mathrm{cpd} / 100 \mathrm{t}$, and explained in detail in [7].

An independent way to measure the ${ }^{210} \mathrm{Bi}$ was suggested in [9], through the measurement of the $\alpha$-emitter ${ }^{210} \mathrm{Po}$ coming from the ${ }^{238} \mathrm{U}$ chain, since it is assumed to be in secular equilibrium with ${ }^{210} \mathrm{Bi}$. However, there are still some residual convective motions in this LPoF even after the thermal insulation campaign. Hence, only a ${ }^{210} \mathrm{Bi}$ upper limit can be obtained by measuring the minimum ${ }^{210} \mathrm{Po}$ rate in the $\mathrm{LPoF}$, and not a measurement of the ${ }^{210} \mathrm{Bi}$ rate. This means that only a lower limit on the $\mathrm{CNO}$ neutrinos can be obtained and not a measurement. However, the significance of the no-CNO hypothesis can still be evaluated, which is the key requirement for providing a direct evidence of $\mathrm{CNO}$ neutrinos.

Two kinds of analyses were performed to extract the CNO significance: a rate only analysis using a simple counting experiment (Section 6), and a rate + shape analysis performed using the Multivariate fit (Section 5). The event sample in Borexino for the CNO analysis is filtered by applying a set of selection criteria [8] that reduce events from residual radioactive impurities, cosmic muons, cosmogenic isotopes, instrumental noise and external $\gamma$-rays. The latter are substantially suppressed by selecting events that occur within an innermost volume of the scintillator (the fiducial volume) as defined by a cut on the reconstructed radius and vertical position $(r<2.8 \mathrm{~m}$ and $-1.8 \mathrm{~m}$ $<z<2.2 \mathrm{~m}$ ). The data are analysed in the electron recoil energy interval between $320 \mathrm{keV}$ and $2,640 \mathrm{keV}$.

\section{The Low Polonium Field}

As discussed previously, the rate of ${ }^{210} \mathrm{Bi}$ decays can be constrained via its link with the ${ }^{210} \mathrm{Po}$ decay rate, with the assumption that this latter term is only supported by in-equilibrium ${ }^{210} \mathrm{~Pb}$ 
decay chain. Data collected by Borexino since its start, however, indicate that an out-of-equilibrium component of ${ }^{210} \mathrm{Po}$ is present in the detector. The source of this component is likely the surface of the inner vessel, from which ${ }^{210} \mathrm{Po}$ is detached into the scintillator. The mean free path of ${ }^{210} \mathrm{Po}$ atoms is calculated to be very small in stable conditions. However, the presence of convective motions in the Borexino scintillator allow ${ }^{210} \mathrm{Po}$ to spread throughout the scintillator volume. Under this conditions, the measured value of ${ }^{210} \mathrm{Po}$ decay rate would be much higher than the ${ }^{210} \mathrm{Bi}$ decay rate, spoiling any possible constraint. To limit convective motions in the scintillator volume, the Borexino Collaboration pursued a long-lasting effort, culminated in the detector thermal insulation in 2015 and the subsequent installation of active temperature controls. This way, ${ }^{210} \mathrm{Po}$ mixing has been strongly suppressed since 2016, leading to the formation of a very clean region around the centre of the detector, called the Low Polonium Field (LPoF). The in-equilibrium ${ }^{210} \mathrm{Po}$ decay rate in the LPoF region can be then measured. However, there might be still some residual contribution of convective ${ }^{210} \mathrm{Po}$ in this region and the measured ${ }^{210} \mathrm{Po}$ rate can therefore only be translated into an upper limit for the ${ }^{210} \mathrm{Bi}$ rate.

The ${ }^{210} \mathrm{Po}$ events in the LPoF have been chosen in the energy range $0.30-0.54 \mathrm{MeV}$ and selected by means of the $\alpha / \beta$ discrimination parameter called the Multi-Layer Perceptron (MLP). A paraboloid equation in $2 \mathrm{D}$, assuming rotational symmetry along the $x-y$ plane, has been used to fit the data. The minimum ${ }^{210} \mathrm{Po}$ rate $\left(R\left({ }^{210} \mathrm{Po}_{\mathrm{min}}\right)\right)$ has been then obtained through the fit function:

$$
\frac{d^{2} R_{P o}}{d\left(\rho^{2}\right) d z}=\left[R\left({ }^{210} P o_{\text {min }}\right) \varepsilon_{E} \varepsilon_{M L P}+R_{\beta}\right]\left(1+\frac{\rho^{2}}{a^{2}}+\frac{\left(z-z_{0}\right)^{2}}{b^{2}}\right) \text {. }
$$

Here, $\rho^{2}=x^{2}+y^{2}, z_{0}$ is the minimum position of the LPoF along the $z$-axis, $a$ and $b$ are shape parameters along the respective axes, $\varepsilon_{E}$ and $\varepsilon_{M L P}$ are the efficiency of the energy and MLP cuts, respectively, applied to select ${ }^{210} \mathrm{Po}$ events, and $R_{\beta}$ is the residual rate of $\beta$ events after the selection of ${ }^{210} \mathrm{Po}$ events. Since the LPoF slowly moves along the $z$-axis due to residual convective motions, data in the LPoF needs to be aligned along the $z$-direction before performing the fit on the full dataset. This has been done by "blindly" aligning the data in the LPoF every month (or every two months) using the centre $z_{0}$ obtained by fitting the data of the previous month. Monthly fits have been performed in large volumes of 70 or 100 ton. After the blind alignment using the centres of every month, the final fit has been performed on the aligned dataset in around 20 ton ( $\sim 5000$ events) using either a simple paraboloid in Equation 2 with four free parameters $\left(R\left({ }^{210} P o_{\text {min }}\right), a, b, z_{0}\right)$ or with more free parameters, depending on the method. The simple paraboloid fit can be performed either as a likelihood fit with ROOT [10] or with the MultiNest Bayesian tool [11]. The assumption of the rotational symmetry has been also verified. In addition to the 2D paraboloid fit, 3D ellipsoidal fits have been also performed with MultiNest without assuming rotational symmetry along the $x-y$ plane, resulting in statistically compatible results. In order to account for the complexity of the LPoF along the $z$-axis, a cubic spline function was implemented along the $z$-axis in equation (2) and the fit was performed with MultiNest. Despite its better fit on the LPoF data, the method was statistically compatible with the simple paraboloid fit and both the methods were finally used for the ${ }^{210} \mathrm{Bi}$ upper limit. In addition to the statistical uncertainty of the fit, the other sources of uncertainties considered in this analysis arise from: (1) the homogeneity of $\beta$-events in the entire 70-ton fiducial volumes, studied in both radial and angluar directions, (2) mass and binning of the data histogram for the fit, and (3) the estimation of the $\beta$-leakage, i.e. $R_{\beta}$ in equation 2 . The final ${ }^{210} \mathrm{Bi}$ upper limit 
obtained through the estimation of the minimum ${ }^{210} \mathrm{Po}$ rate in the LPoF of Borexino, including both statistical and systematic contribution, is $R\left({ }^{210} \mathrm{Bi}\right) \leq(11.5 \pm 1.3) \mathrm{cpd} / 100$ ton.

\section{Multivariate spectral fit}

CNO neutrinos are disentangled from residual backgrounds using a multivariate analysis, which includes the energy and radial distributions of the events surviving the selection. Data are split into two complementary datasets: the threefold-coincidence (TFC)-subtracted spectrum, in which ${ }^{11} \mathrm{C}$ is selectively filtered out using the muon-neutron-positron threefold coincidence algorithm [7, 8] and the TFC-tagged spectrum, enriched in ${ }^{11} \mathrm{C}$. The resulting total likelihood function is simply a product of the functions corresponding to each spectrum is as follows:

$$
\mathcal{L}_{M V}(\vec{\theta})=\mathcal{L}_{T F C-t a g}(\vec{\theta}) \cdot \mathcal{L}_{T F C-s u b}(\vec{\theta}) \cdot \mathcal{L}_{R D}(\vec{\theta}) .
$$

The reference shapes for signal and backgrounds used in the fit are obtained through a complete Geant4-based Monte Carlo code, which simulates all physics processes occurring in the scintillator, including energy deposition, photon emission, propagation, and detection, generation and processing of the electronic signal. The simulation takes into account the evolution in time of the detector response and produces data that are reconstructed and selected following the same pipeline of real data. In addition to the energy shape, other information is exploited to help the fit to disentangle the signal from background: the ${ }^{11} \mathrm{C} \beta^{+}$events are tagged by TFC, and contributions from the external backgrounds $\left({ }^{208} \mathrm{Tl},{ }^{214} \mathrm{Bi}\right.$ and $\left.{ }^{40} \mathrm{~K}\right)$ are further constrained due to their radial distribution. The pep and the ${ }^{210} \mathrm{Bi}$ components are constrained using symmetric Gaussian and half-Gaussian (upper limit) terms in the fit, with the values discussed in Sections 3 and 4, respectively. The other components are left to vary. The $p$-value of the fit is 0.3 , demonstrating fair agreement between data and the underlying fit model. A non-zero CNO neutrino rate is clearly better suited to the fit, as shown in the log-likelihood profile of Figure 2 (dashed black curve). The final CNO neutrino rate obtained from this analysis is $7.2_{-1.7}^{+3.0} \mathrm{cpd} / 100 \mathrm{t}$. The effect of the following sources of deformations were included in the systematic uncertainty evaluation: (1) Energy response function (0.9\%), (2) deformations of the ${ }^{11} \mathrm{C}$ spectral shape (2.3\%), and (3) spectral shape of ${ }^{210} \mathrm{Bi}(18 \%)$. From this Monte Carlo study, the CNO systematic error due to a mismatch between real and simulated PDFs was found to be ${ }_{-0.6}^{+0.5} \mathrm{cpd}$ per $100 \mathrm{t}$.

\section{Counting analysis}

Complementary to the rate + shape analysis that was be explained in the previous section, a pure counting experiment can be performed in a particular Region of Interest (ROI), where CNO, pep, and ${ }^{210} \mathrm{Bi}$ are dominant. The $\mathrm{CNO}$ rate is calculated as the difference between the total number of events detected in the ROI and that of the backgrounds, evaluated in an independent way. The chosen ROI, $780-885 \mathrm{keV}$, is obtained optimizing the CNO signal-to-background ratio. An advantage of this method is that, in the ROI, some of the backgrounds that affect the multivariate analysis (such as ${ }^{85} \mathrm{Kr}$ and ${ }^{210} \mathrm{Po}$ ) are not present or contribute less than $2 \%$ (for example, external backgrounds). The count rate is dominated by $\mathrm{CNO}$, pep and ${ }^{210} \mathrm{Bi}(80 \%)$, with smaller contributions from ${ }^{7} \mathrm{Be}$ neutrinos and residual ${ }^{11} \mathrm{C}(18 \%)$. The rate of pep neutrinos and ${ }^{210} \mathrm{Bi}$ are constrained to the same 


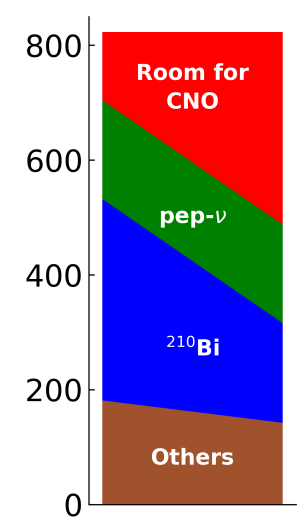

Events in the ROI

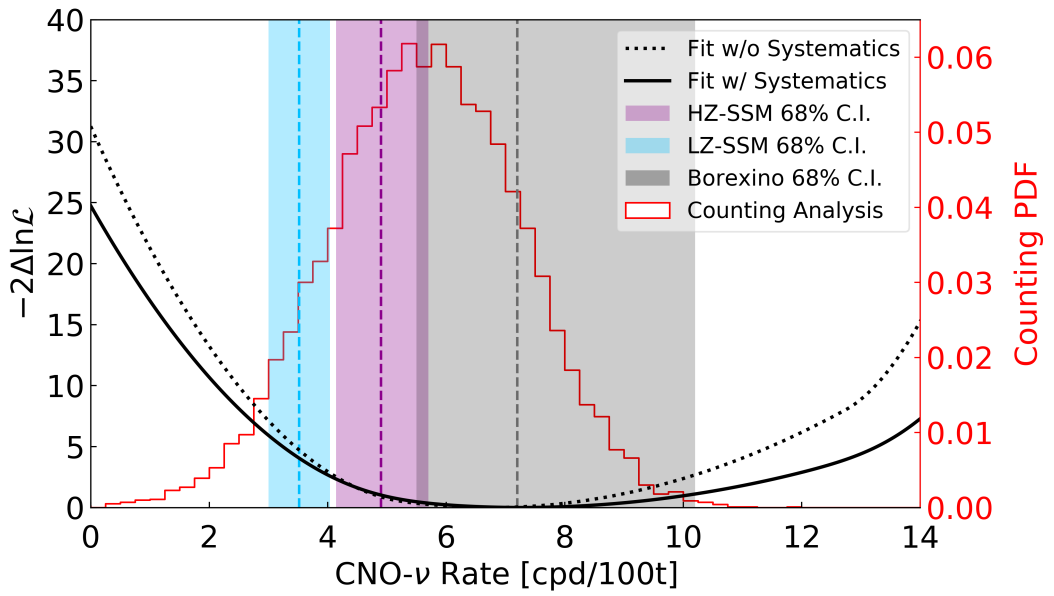

Figure 2: Left: Counting analysis. The vertical axis is the number of events allowed by the data for CNO neutrinos and backgrounds in the ROI; on the left, the CNO signal is minimum and backgrounds are maximum, while on the right, $\mathrm{CNO}$ is maximum and backgrounds are minimum. It is clear from this figure that CNO cannot be zero. Right: CNO neutrino rate negative log-likelihood profile directly from the multivariate fit (dashed black line) and after folding in the systematic uncertainties (black solid line). Histogram in red: $\mathrm{CNO}$ neutrino rate obtained from the counting analysis. Finally, the blue, violet, and grey vertical bands show $68 \%$ confidence intervals (C.I.) for the LZ and HZ SSM predictions and the Borexino result (corresponding to black solid-line log-likelihood profile), respectively. Taken from [7].

values used in the multivariate fit. Note that whereas in the spectral fit the ${ }^{210} \mathrm{Bi}$ rate is left free to vary between 0 up to $11.5 \pm 1.3 \mathrm{cpd}$ per $100 \mathrm{t}$ (the upper limit determined in the LPoF analysis), the counting analysis conservatively constrains it to the maximum value with a Gaussian error of 1.3 cpd per $100 \mathrm{t}$. The ${ }^{7} \mathrm{Be}$ neutrino rate is sampled uniformly between the low-metallicity $(43.7 \pm 2.5$ cpd per $100 \mathrm{t})$ and the high-metallicity $(47.9 \pm 2.8 \mathrm{cpd}$ per $100 \mathrm{t})$ values predicted by the Standard Solar Model with $1 \sigma$ error, whereas the ${ }^{11} \mathrm{C}$ rate is obtained from the average Borexino PhaseII results with an additional conservative error of $10 \%$ derived from uncertainties on the energy scale (quenching of the $1 \mathrm{MeV}$ annihilation $\gamma$-rays). The $\mathrm{CNO}$ rate is obtained by subtracting all background contributions defined above and by propagating the uncertainties by randomly sampling their rates from Gaussian distributions with proper widths. Note that the uncertainty related to the energy response (which affects the percentage of the spectrum of each component falling in the ROI) also contributes to the total error associated with the count rate of each species. The systematic uncertainties described in the previous section were also used for this analysis. The CNO rate obtained with this method is demonstrated by the red histogram in Figure 2. The mean value and width of the distribution are $5.6 \pm 1.6 \mathrm{cpd}$ per $100 \mathrm{t}$, confirming the presence of $\mathrm{CNO}$ at $3.5 \sigma$ level.

\section{Results}

A frequentist hypothesis test [12] was performed to extract the significance for the rejection of the null hypothesis of the CNO signal in the Sun. 13.8 million toy MC datasets including systematic uncertainty on MC shapes were fitted using the MV fit and a $q_{0}$ distribution was obtained. Using the $q_{o b s}=30.05$ value obtained from the MV fit, a $p$-value was obtained by calculating the integral of the $q_{0}$ curve that falls after $q_{o b s}$. This resulted in the rejection of the null-hypothesis of the CNO 
signal with greater than $5 \sigma$ significance with $99 \%$ C.L. This is compatible with the significance of 5.1 $\sigma$ extracted from the log-likelihood profile obtained from the MV fit as shown in Figure 2 (black solid line). The systematic uncertainty obtained from the MC datasets was included for this by smearing the log-likelihood profile with a symmetric Gaussian. The $68 \%$ C.I. obtained from the log-likelihood profile in Figure 2 (shaded grey area), is [5.5-10.2] cpd/100t. The neutrino rate translates to a flux of 7.5 $5_{-2.0}^{+3.0} \times 10^{8} \mathrm{~cm}^{-2} \mathrm{~s}^{-1}$ on Earth [7], assuming MSW conversion in matter, neutrino oscillation parameters from, and a density of electrons of $(3.307 \pm 0.015) \times 10^{31} e^{-}$per $100 \mathrm{t}$ in the LS. Thus, Borexino provided the first direct experimental evidence for the presence of CNO cycle solar neutrinos in the Sun.

\section{References}

[1] C. von Weizsäcker, "Über elementumwandlungen in innern der sterne ii," Phyikalische Zeitschrift, vol. 39, p. 633, 1938.

[2] H. Bethe, "Energy production in stars," Phys. Rev., vol. 55, pp. 434-456, 1939.

[3] N. Vinyoles, A. M. Serenelli, F. L. Villante, S. Basu, J. Bergström, M. Gonzalez-Garcia, M. Maltoni, C. Peña-Garay, and N. Song, "A new Generation of Standard Solar Models," Astrophys. J., vol. 835, no. 2, p. 202, 2017.

[4] N. Grevesse and A. J. Sauval, "Standard Solar Composition,” Space Sci. Rev., vol. 85, pp. 161174, 1998.

[5] M. Asplund, N. Grevesse, A. Sauval, and P. Scott, "The chemical composition of the Sun," Ann. Rev. Astron. Astrophys., vol. 47, pp. 481-522, 2009.

[6] G. Alimonti et al., "The Borexino detector at the Laboratori Nazionali del Gran Sasso," Nucl. Instrum. Meth., vol. A600, pp. 568-593, 2009.

[7] M. Agostini et al., "Experimental evidence of neutrinos produced in the CNO fusion cycle in the Sun," Nature, vol. 587, pp. 577-582, 2020.

[8] M. Agostini et al., "Comprehensive measurement of pp-chain solar neutrinos," Nature, vol. 562, no. 7728, pp. 505-510, 2018.

[9] F. Villante, A. Ianni, F. Lombardi, G. Pagliaroli, and F. Vissani, “A Step toward CNO solar neutrinos detection in liquid scintillators," Phys. Lett. B, vol. 701, pp. 336-341, 2011.

[10] R. Brun and F. Rademakers, "Root - an object oriented data analysis framework," Nuclear Instruments \& Methods in Physics Research A, vol. 389, no. 08, pp. 81-86, 1997.

[11] F. Feroz and M. Hobson, "Multimodal nested sampling: an efficient and robust alternative to Markov Chain Monte Carlo methods for astronomical data analyses," Monthly Notices of the Royal Astronomical Society, vol. 384, pp. 449-463, 012008.

[12] G. Cowan, K. Cranmer, E. Gross, and O. Vitells, "Asymptotic formulae for likelihoodbased tests of new physics," Eur. Phys. J., vol. C71, p. 1554, 2011. [Erratum: Eur. Phys. J.C73,2501(2013)]. 


\section{Full Authors List: BorexinoCollaboration}

M. Agostini ${ }^{2,3}$, K. Altenmüller ${ }^{3}$, S. Appel ${ }^{3}$, V. Atroshchenko ${ }^{4}$, Z. Bagdasarian ${ }^{1,1}$, D. Basilico ${ }^{5}$, G. Bellini $^{5}$, J.Benziger ${ }^{6}$, R.

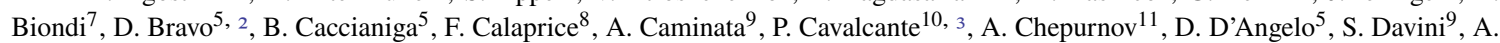
Derbin $^{12}$, A. Di Giacinto ${ }^{7}$, V. Di Marcello ${ }^{7}$, X.F. Ding ${ }^{8}$, A. Di Ludovico ${ }^{8}$, L. Di Noto ${ }^{9}$, I. Drachnev ${ }^{12}$, A. Formozov ${ }^{13,6}$, D. Franco ${ }^{14}$, C. Galbiati ${ }^{8,15}$, C. Ghiano ${ }^{7}$, M. Giammarchi ${ }^{5}$, A. Goretti ${ }^{8, c}$, A. S. Göttel ${ }^{1,16}$, M. Gromov ${ }^{11,14}$, D. Guffanti ${ }^{17}$, Aldo Ianni ${ }^{7}$, Andrea Ianni $^{8}$, A. Jany ${ }^{18}$, D. Jeschke ${ }^{3}$, V. Kobychev ${ }^{19}$, G.Korga ${ }^{20,21}$, S. Kumaran ${ }^{1,16}$, M. Laubenstein ${ }^{7}$, E. Litvinovich ${ }^{4,22}$, P. Lombardi ${ }^{5}$, I. Lomskaya ${ }^{12}$, L. Ludhova ${ }^{1,16}$, G. Lukyanchenko ${ }^{4}$, L. Lukyanchenko ${ }^{4}$, I. Machulin ${ }^{4,22}$, J. Martyn ${ }^{17}$, E. Meroni ${ }^{5}$, M. Meyer ${ }^{23}$, L. Miramonti $^{5}$, M. Misiaszek ${ }^{18}$, V. Muratova ${ }^{12}$, B. Neumair ${ }^{3}$, M. Nieslony ${ }^{17}$, R. Nugmanov ${ }^{4,22}$, L. Oberauer $^{3}$, V. Orekhov ${ }^{17}$, F. Ortica ${ }^{24}$, M. Pallavicini ${ }^{9}$, L. Papp ${ }^{3}$, L. Pelicci ${ }^{1,16}$, Ö. Penek ${ }^{1,16}$, L. Pietrofaccia ${ }^{8}$, N. Pilipenko ${ }^{12}$, A. Pocar ${ }^{25}$, G. Raikov ${ }^{4}$, M. T. Ranalli ${ }^{7}$, G. Ranucci ${ }^{5}$, A. Razeto ${ }^{7}$, A. Re ${ }^{5}$, M. Redchuk ${ }^{1,16,4}$, A. Romani ${ }^{24}$, N. Rossi ${ }^{7}$, S. Schönert ${ }^{3}$, D. Semenov ${ }^{12}$, G. Settanta ${ }^{1,2}$, M. $^{2}$ Skorokhvatov $^{4,22}$, A. Singhal ${ }^{1,16}$, O. Smirnov ${ }^{13}$, A. Sotnikov ${ }^{13}$, Y. Suvorov ${ }^{4,8,5}$, R. Tartagliaa ${ }^{7}$, G. Testera ${ }^{9}$, J. Thurn ${ }^{23}$, E. Unzhakov ${ }^{12}$, F. L. Villante ${ }^{7,26}$, A. Vishneva ${ }^{13}$, R. B. Vogelaar ${ }^{10}$, F. von Feilitzsch ${ }^{3}$, M. Wojcik ${ }^{18}$, M. Wurm ${ }^{17}$, S. Zavatarelli ${ }^{9}$, K. Zuber $^{23}$, G. Zuzel ${ }^{18}$.

${ }^{1}$ Institut für Kernphysik, Forschungszentrum Jülich, 52425 Jülich, Germany

${ }^{2}$ Department of Physics and Astronomy, University College London, London, UK

${ }^{3}$ Physik-Department, Technische Universität München, 85748 Garching, Germany

${ }^{4}$ National Research Centre Kurchatov Institute, 123182 Moscow, Russia

${ }^{5}$ Dipartimento di Fisica, Università degli Studi e INFN, 20133 Milano, Italy

${ }^{6}$ Chemical Engineering Department, Princeton University, Princeton, NJ 08544, USA

${ }^{7}$ INFN Laboratori Nazionali del Gran Sasso, 67010 Assergi (AQ), Italy

${ }^{8}$ Physics Department, Princeton University, Princeton, NJ 08544, USA

${ }^{9}$ Dipartimento di Fisica, Università degli Studi e INFN, 16146 Genova, Italy

${ }^{10}$ Physics Department, Virginia Polytechnic Institute and State University, Blacksburg, VA 24061, USA

${ }^{11}$ Lomonosov Moscow State University Skobeltsyn Institute of Nuclear Physics, 119234 Moscow, Russia

${ }^{12}$ St. Petersburg Nuclear Physics Institute NRC Kurchatov Institute, 188350 Gatchina, Russia

${ }^{13}$ Joint Institute for Nuclear Research, 141980 Dubna, Russia

${ }^{14}$ AstroParticule et Cosmologie, Université Paris Diderot, CNRS/IN2P3, CEA/IRFU, Observatoire de Paris, Sorbonne Paris Cité, 75205 Paris Cedex 13, France

${ }^{15}$ Gran Sasso Science Institute, 67100 L'Aquila, Italy

${ }^{16}$ RWTH Aachen University, 52062 Aachen, Germany

${ }^{17}$ Institute of Physics and Excellence Cluster PRISMA+, Johannes Gutenberg-Universität Mainz, 55099 Mainz, Germany

${ }^{18}$ M. Smoluchowski Institute of Physics, Jagiellonian University, 30348 Krakow, Poland

${ }^{19} \mathrm{Kiev}$ Institute for Nuclear Research, $03680 \mathrm{Kiev}$, Ukraine

${ }^{20}$ Department of Physics, Royal Holloway, University of London, Department of Physics, School of Engineering, Physical and Mathematical Sciences, Egham, Surrey, TW20 OEX, UK

${ }^{21}$ Institute of Nuclear Research (Atomki), Debrecen, Hungary

${ }^{22}$ National Research Nuclear University MEPhI (Moscow Engineering Physics Institute), 115409 Moscow, Russia

${ }^{23}$ Department of Physics, Technische Universität Dresden, 01062 Dresden, Germany

${ }^{24}$ Dipartimento di Chimica, Biologia e Biotecnologie, Università degli Studi e INFN, 06123 Perugia, Italy

${ }^{25}$ Amherst Center for Fundamental Interactions and Physics Department, University of Massachusetts, Amherst, MA 01003, USA

${ }^{26}$ Dipartimento di Scienze Fisiche e Chimiche, Università dell'Aquila, 67100 L'Aquila, Italy

${ }^{a}$ Present address: University of California, Berkeley, Department of Physics, CA 94720, Berkeley, USA

${ }^{b}$ Present address: Universidad Autónoma de Madrid, Ciudad Universitaria de Cantoblanco, 28049 Madrid, Spain

${ }^{c}$ Present address: INFN Laboratori Nazionali del Gran Sasso, 67010 Assergi (AQ), Italy

${ }^{d}$ Present address: Dipartimento di Fisica e Astronomia dell'Università di Padova and INFN Sezione di Padova, Padova, Italy

${ }^{e}$ Present address: Dipartimento di Fisica, Università degli Studi Federico II e INFN, 80126 Napoli, Italy 